\title{
The Optimization of Multiquadric Function in Reverse Engineering
}

\author{
Dejun Liu \\ Mathematics and Physics Department of Officers College of PAP \\ Chengdu, China \\ e-mail:liudejun2365tian@126.com
}

Keywords: reverse engineering; MQ function; Node proportion; scale parameter.

Abstract: Reconstructing surface from the large scattered data is a ubiquitous problem in reverse engineering. Implicit surfaces reconstruction methods using Multiquadric function have recently attracted attention for its high precision and continuous order. This paper get the relative efficient proportion of interpolation nodes and the range of the scale parameter in the Multiquadric function Through the mathematical experiments and deviation analysis.

\section{Introduction}

Reconstructing surface from the large scattered data is a ubiquitous problem in reverse engineering. Multiquadric function(MQ) interpolation is a stable and accurate method of solving multivariate scattered data interpolation. However MQ interpolation with scattered data points has seldom been adopted in the mathematics experiment to get the proportion of interpolation nodes and the range of the scale parameter. This method was introduced by Hardy in 1971, when he meets the problems of fitting curve with scattered data in drawing a contour line. Hardy proposed a new radial basis function named MQ function which can be expressed as

$$
\sqrt{\left\|\mathrm{X}-X_{i}\right\|^{2}+r^{2}}
$$

With this basis function, they constructed the dual function surface and received some good results ${ }^{[1]}$. Later, Frank ${ }^{[2]}$ compared numbers of interpolation methods with scattered data and considered that the results of Multiquadric (MQ) method is the most accurate one. Then this method is widely and successfully used in the meteorology, environment, and marine et.al. Micchelli studied the density, interpolation and convergence of MQ function, which provide the theoretical background for radial basis function class in Scattered Data Interpolation ${ }^{[3]}$. In recent years, several studies were developed in the literature, some discuss the number of coefficient condition of linear equations and some improve interpolation functions, but seldom identified the efficient proportion of interpolation nodes. As we know, the improper selection of the proportion of interpolation nodes will cause significant errors. Therefore, We present a deeper research by theoretical and experiments, and get a better range of the proportion of interpolation nodes.

The so-called MQ function interpolation is finding the function which has the form as follow

$$
S(\mathrm{X})=\sum_{i=1}^{\mathrm{N}} c_{i} \phi\left(\left\|X-X_{i}\right\|\right), X_{i} \in R^{d}
$$

where $\quad \phi\left(\left\|X-X_{i}\right\|\right)=\sqrt{\left\|\mathrm{X}-X_{i}\right\|^{2}+r^{2}}$.

For a given data $\left\{\mathrm{X}_{\mathrm{i}}, f \mathrm{i}\right\}, \mathrm{X}_{i} \in R^{d}, f_{i} \in R, i=1,2, \cdots, n$

Then

$$
\begin{gathered}
S\left(X_{i}\right)=f_{i}, i=1,2, \cdots, \mathrm{N} \\
\cdot \dot{X}_{1}, \cdots, X_{N} \in R^{d} \quad \mathrm{c}_{1}, c_{2}, \cdots, c_{N} \in R
\end{gathered}
$$

If the data is given, the unknown weight coefficients linear equations can be obtained from (1). 
$\left[\begin{array}{cccc}\phi_{11} & \phi_{12} & \cdots & \phi_{1 \mathrm{~N}} \\ \phi_{21} & \phi_{22} & \cdots & \phi_{2 \mathrm{~N}} \\ \vdots & \vdots & \vdots & \vdots \\ \phi_{\mathrm{N} 1} & \phi_{\mathrm{N} 2} & \cdots & \phi_{\mathrm{NN}}\end{array}\right]\left[\begin{array}{c}\mathrm{c}_{1} \\ c_{2} \\ \vdots \\ c_{N}\end{array}\right]=\left[\begin{array}{c}f_{1} \\ f_{2} \\ \vdots \\ f_{N}\end{array}\right]$

where $\phi_{\mathrm{ij}}=\phi\left(\left\|X_{i}-X_{j}\right\|\right), i, j=1,2, \cdots, N$.If the first matrix on the left of (2) is nonsingular, its inverse matrix exists, then we can solve the coefficient vector $C$ from (2), and get the specific form of interpolation functions ${ }^{[4]}$.

\section{Theoretical presentation}

In this text we primary considerate the MQ function $\phi(\|\bullet\|)=\sqrt{c+\|X\|^{2}}$ to interpolate sphere in three-dimensional space. By a great deal experiment data we will get the relative efficient proportion of interpolation nodes in the MQ function $\phi(\|\bullet\|)=\sqrt{c+\|X\|^{2}}$ through the experiments and error analysis.

During the experiment, In order to avoid trivial solution of the linear equations corresponded to the interpolation matrix; we must construct the constraint points out the surface. We use the Normal vector constraint and boundary constraint method introduced by Turk et al $^{[5]}$ The interpolation points on the surface form a set of boundary constraint and the value of interpolation function at these points is 0 ; And the point out the surface form a set of normal vector constraint and the value of interpolation function at these points is $\pm 1^{[6]}$. The spherical surface parameter equation is:

$$
\left\{\begin{array}{l}
\mathrm{x}=R \cos \theta \cos \varphi \\
y=R \cos \theta \sin \varphi \\
z=R \sin \theta
\end{array}-\frac{\pi}{2} \leq \theta \leq \frac{\pi}{2}, 0 \leq \varphi<2 \pi\right.
$$

It is not hard to see from the spherical surface parameter equation that the spherical surface normal vector at each point is

$$
\overrightarrow{\mathrm{n}}=(R \cos \theta \cos \varphi, R \cos \theta \sin \varphi, \quad R \sin \theta),-\frac{\pi}{2} \leq \theta \leq \frac{\pi}{2}, 0 \leq \varphi \leq 2 \pi
$$

Without loss of generality ,Here We use $\mathrm{X}_{i}+k n_{i}$ and $\mathrm{X}_{i}-k n_{i}$ construct the constraint point set of the outside and inside the Spherical surface. The value of interpolation function at these points is 1 and -1 respectively. Where $\mathrm{k}>0$, ni is the normal vector correspond to $\mathrm{Xi}$. In this experiment we only take the constraint point outside the surface and the value of interpolation function at these points is 1 . Here are the basic processes of experimental procedure:

Step1: Get the specific coordinates of the nodes through the program;

Step2: Calculate the undetermined coefficients by the specific interpolation nodes and interpolation functions and obtain the specific form of interpolation matrix;

Step3: Find the solution of the interpolation Matrix with Matrix Laboratory package, then gets the power factor value $\mathrm{c}_{i}, i=0,1, \cdots, N$ and the hypersurface;

Step4: Distil the Zero iso-surface and draw graphics with graphics software;

Step5: Calculate the Root Mean Square (RMS ) errors;

Step6: Change the proportion of interpolate nodes and restart from Step1.

Here we use the interpolation function $\phi(\|\bullet\|)=\sqrt{c+\|X\|^{2}}$, select 1423 interpolation nodes and set $k=0.5$. For analysising the effect of the number of interpolation nodes which outside the surface, some representative experimental data are fitted in the Table1: 
Table 1 (The Root Mean Square errors for different interpolate nodes)

\begin{tabular}{|l|l|l|l|l|l|l|}
\hline $\mathrm{n}$ & 700 & 650 & 630 & 620 & 600 & 580 \\
\hline$e$ & 0.1401 & 0.1352 & 0.1331 & 0.1320 & 0.1299 & 0.1277 \\
\hline $\mathrm{n}$ & 550 & 500 & 410 & 400 & 353 & 323 \\
\hline$e$ & 0.1243 & 0.1185 & 0.1073 & 0.1060 & 0.0996 & 0.0953 \\
\hline $\mathrm{n}$ & 303 & 223 & 160 & 123 & 23 & 13 \\
\hline$e$ & 0.0923 & 0.0792 & 0.0671 & 0.0588 & 0.0254 & 0.0191 \\
\hline
\end{tabular}

We can get multiple type of deviation ${ }^{[7,8]}$ in the literature. but our study is only focus on comparing the data, so we choose the root-mean-square error, which has the simple form and can increase the experimental efficiency. As we know, the effect of the constraint points outside the surface to the fitting accuracy is less than it on the surface. The experimental data in Table 1 is also preliminary confirm this conclusion, that the interpolation result is better for less number of constraint points outside the surface. We can observe the following interpolation image from the experiment to draw a conclusion.

As Figure 1 shows, it is easy to see that as the number of constraint points outside the surface increased the interpolation result will be worse, and as the number of constraint points outside the surface decreased the interpolation result will be better. However this rule is not absolute, it will lead to solution instability if the number of points outside the surface is too little,thus affecting the interpolation results. As Shown in Figure 1,when there are 13 constraint points away the surface, there are a large number of "sawtooth" around the graphics, caused by so few constraint points outside the surface choosed in the experiment. It can be obtained by more experiments that the interpolation result would be better if the proportion of the constraint point outside the surface is between $9 \%$ to $14 \%$.
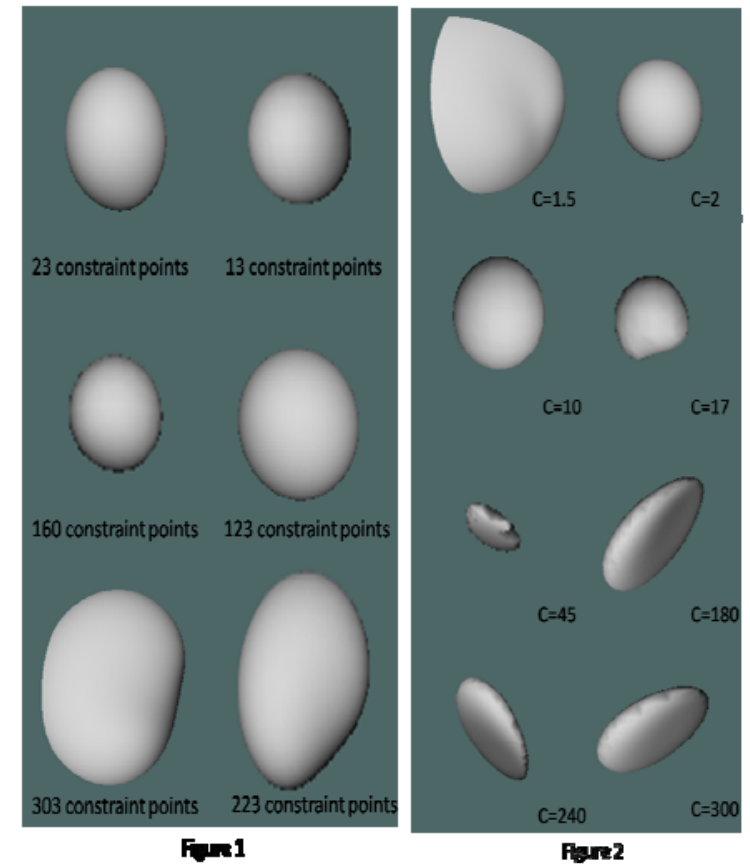

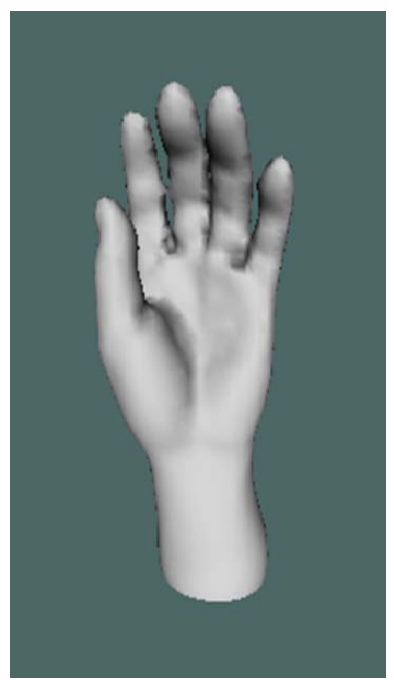

Figure 3

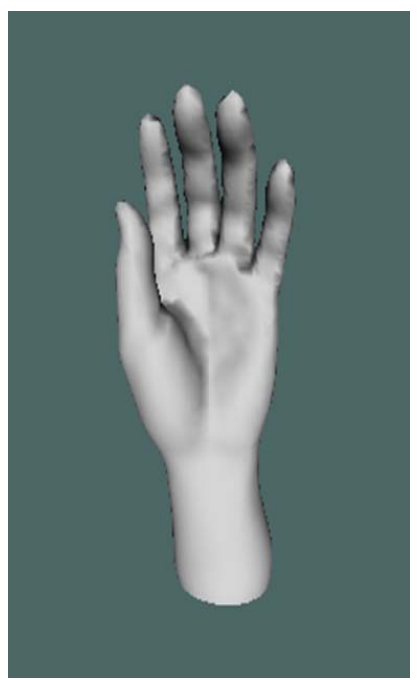

Figure 4

Now we analyses the effect of the interpolation results caused by the scale parameter $\mathrm{C}$ in the function $\phi(\|\bullet\|)=\sqrt{c+\|X\|^{2}}$. In order to facilitate observation we take 1300 constraint points on the sphere of radius $1\left\{\mathrm{X}_{i} \in R^{3}, i=1,2, \cdots, 1200\right\}$ and 123 constraint points outside the surface $\left\{\mathrm{X}_{j} \in R^{3}, j=1301,1202, \cdots, 1423\right\}, k=0.5$. A large amount of data obtained by experiment, we take the representative data in the table2 : 
Table 2 ( Root Mean Square (RMS) : e, $\phi(\|\bullet\|)=\sqrt{c+\|X\|^{2}}$ )

\begin{tabular}{|l|l|l|l|l|l|l|}
\hline c & \multicolumn{1}{|c|}{2} & \multicolumn{1}{c|}{4} & \multicolumn{1}{c|}{6} & \multicolumn{1}{c|}{7} & \multicolumn{1}{c|}{9} \\
\hline e & 0.058794 & 0.058790 & 0.058790 & 0.058797 & 0.058785 & 0.058795 \\
\hline c & 11 & 12 & 13 & 15 & 20 & 40 \\
\hline e & 0.0588801 & 0.058260 & 0.062715 & 0.058838 & 0.058791 & 0.059200 \\
\hline c & 70 & 95 & 130 & 160 & 180 & 200 \\
\hline e & 0.058903 & 0.059001 & 0.059265 & 0.060192 & 0.062387 & 0.05994 \\
\hline c & 220 & 240 & 270 & 300 & 350 & 400 \\
\hline e & 0.059255 & 0.059556 & 0.060069 & 0.059085 & 0.059755 & 0.059122 \\
\hline
\end{tabular}

From table 2, we can see that it shows certain regularity. In order to draw the broader conclusion, we can observe the following interpolation image obtained by the experiments.

From Figure 2, it is easy to see that the interpolation error is smaller when the scale parameter varies between 2 to 10, and the interpolation deviation is larger when the scale parameter is greater than 10. In addition, we can found that the solution of linear equations is often instability when the scale parameter less than 1 . Therefore, the values of scale parameter in the interpolation between 2 to 10 are relatively better.

In order to verify the conclusion obtained above, we make a hand model surface reconstruction experiment as shown in Figure 3 and Figure 4. We used 1338 scattered data after filtration, Here $\mathrm{c}=2 k=0.04$. Figure 3 is the result of reconstruction in which the number of points outside the surface is 360 , it is easy to see the " adhesion " phenomenon.Figure 4 is the better reconstructed surface in which 140 points outside the surface were selected in experiment. We found that it cannot be reconstructed with high precision if the number of points outside the surface or the values of scale parameter are out of the range obtained by the former experiments.

\section{Conclusions}

Overall, based on massive experimental data and deviation analysis, we can obtain the better MQ interpolation results and reconstructed images when the proportion of the points outside the surface range from $10 \%-14 \%$. the values of scale parameter in the interpolation between 2 to 10 are relatively better. With those parameters, we made a hand model surface reconstruction experiment and obtained a good result. Therefore the conclusion is also valid for other mathematics experiments.

\section{References}

[1] R.Hardy. Multiquadric equations of topography and other irregular surfaces. Geophysical Reseach,1971,76:1905-1915.

[2] Frank R. Scattered data interpolation: tests of some methods[J].Math Comp,1982,38:191-200.

[3] Micchelli C. Interpolation of scattered data: distance matrix and conditionally positive definite functions[J].Constr,Approx.1986,2:11-12.

[4] Lixia Guo. Three-dimensional reconstruction technique researching based on RBF point cloud data[D]. Master thesis in Northwestern University, 2006.

[5] G.Turk and J.o'Brien.Modeling with implicit surfaces that interpolate[J].ACM Transactions on Graphics,2002,21:855-873

[6] Xinwei Du.Reseach on Technology of Surface Reconstruction Using Radial Basis Functions in Reverse Engineering [D]. Master thesis in Jilin University, 2006.

[7] Schaback R. Error estimates and condition numbers for radial basis function interpolation. A dv.Comput.Math.1995,3:251-264. 
[8] Wu Z, Schaback R. Local error estimates for radial basis function interpolation of scattered data[J]. IMA J . of Numerical Analysis , 1993 , $13: 13-27$. 